\title{
Impact of EGFR amplification on survival of patients with EGFR exon 20 insertion-positive non-small cell lung cancer
}

\author{
Xin Gao ${ }^{1,2}$, Xue-Wu Wei ${ }^{2}$, Ming-Ying Zheng ${ }^{2}$, Zhi-Hong Chen ${ }^{2}$, Xu-Chao Zhang ${ }^{2}$, Wen-Zhao Zhong ${ }^{2}$, \\ Jin-Ji Yang ${ }^{2}$, Yi-Long $\mathrm{Wu}^{2}$, Qing Zhou ${ }^{1,2}$ \\ ${ }^{1}$ The Second School of Clinical Medicine, Southern Medical University, Guangzhou, China; ${ }^{2}$ Guangdong Lung Cancer Institute, Guangdong \\ Provincial Key Laboratory of Translational Medicine in Lung Cancer, Guangdong Provincial People's Hospital \& Guangdong Academy of Medical \\ Sciences, School of Medicine, South China University of Technology, Guangzhou, China \\ Contributions: (I) Conception and design: X Gao, Q Zhou, YL Wu; (II) Administrative support: ZH Chen; (III) Provision of study materials or \\ patients: MY Zheng, XC Zhang, JJ Yang, WZ Zhong; (IV) Collection and assembly of data: X Gao, XW Wei; (V) Data analysis and interpretation: X \\ Gao, Q Zhou, YL Wu; (VI) Manuscript writing: All authors; (VII) Final approval of manuscript: All authors. \\ Correspondence to: Qing Zhou. Guangdong Lung Cancer Institute, Guangdong Provincial Key Laboratory of Translational Medicine in Lung Cancer, \\ Guangdong Provincial People's Hospital \& Guangdong Academy of Medical Sciences, 106 Zhongshan 2nd Road, Guangzhou 510080, China. \\ Email: gzzhouqing@126.com.
}

Background: Epidermal growth factor receptor (EGFR) exon 20 insertion (EGFR ex20ins) is a common mutation in non-small cell lung cancer (NSCLC). Patients with EGFR ex20ins generally respond poor to EGFR-tyrosine kinase inhibitors (EGFR-TKIs). EGFR ex20ins are often co-occurring with EGFR amplification. However, the impact of EGFR amplification on the survival of patients with EGFR ex20ins mutations has not been determined.

Methods: This is an observational longitudinal cohort study. A prospectively managed database included consecutive treatment-naïve adult patients with advanced NSCLC and EGFR ex20ins confirmed by nextgeneration sequencing (NGS) at Guangdong Provincial People's Hospital between November 2017 and February 2019. The participants were enrolled from the database and extracted their clinical characteristics, treatment and clinical outcomes. NGS was used to establish whether EGFR amplification was present in tumor tissue. Overall survival (OS) and progression-free survival (PFS) were compared between EGFR amplification and non-EGFR amplification groups using the Kaplan-Meier method and log-rank test. Subgroup analyses were performed based on the treatment used (EGFR-TKI or chemotherapy).

Results: Fifteen different EGFR ex20ins mutation subtypes were identified in the 39 patients included in the analysis, and the most common subtypes were p.A767_D770dup (25.6\%), p.S768_D770dup (23.1\%) and p.N771_H773dup (10.3\%). Among 31 patients with EGFR ex20ins mutations and NGS data for tumor tissue, EGFR amplification was identified in 12 patients $(38.7 \%)$ and there were no significant differences in clinical characteristics. Among 26 patients, there were no significant differences between the EGFR amplification $(\mathrm{n}=11)$ and non-EGFR amplification $(\mathrm{n}=15)$ groups in median OS (715 vs. 452 days, $\mathrm{P}=0.912)$. Among 20 patients administered chemotherapy, there were no significant differences between the EGFR amplification and non-EGFR amplification groups in median PFS (206 vs. 112 days, $\mathrm{P}=0.425)$. Among 24 patients administered an EGFR-TKI, median PFS was longer in the non-EGFR amplification group than in the EGFR amplification group (110 vs. 31 days, $\mathrm{P}=0.030$ ).

Conclusions: There is a tendency that EGFR amplification might be a poor predictor in EGFR ex20inspositive NSCLC patients treated with EGFR-TKIs.

Keywords: Non-small cell lung cancer (NSCLC); epidermal growth factor receptor; exon 20; gene insertion; gene amplification

Submitted Apr 09, 2020. Accepted for publication Aug 24, 2020.

doi: $10.21037 /$ jtd-20-1630

View this article at: http://dx.doi.org/10.21037/jtd-20-1630

(c) Journal of Thoracic Disease. All rights reserved. 


\section{Introduction}

Lung cancer is one of the most common malignant diseases worldwide and a major cause of cancer-related death $(1,2)$. Around $85 \%$ of all lung cancers are non-small cell lung cancers (NSCLCs), among which adenocarcinoma and squamous cell carcinoma (SCC) are the most common types $(3,4)$. During the past decade, the development of new technologies such as next-generation sequencing (NGS) has enhanced our understanding of how the genetic characteristics of NSCLC influence its pathogenesis and drug-resistance (5). The use of modern molecular biology tools to study the genetic changes underlying NSCLC has allowed us to embark on an era of precision medicine, and as a result the one-size-fits-all therapeutic model has been abandoned. It is now known that mutations in the gene encoding the epidermal growth factor receptor (EGFR) promote tumor proliferation and metastasis, and the use of EGFR-tyrosine kinase inhibitors (EGFR-TKIs) as a targeted therapy has markedly improved the management of patients with NSCLC (5-7).

In general EGFR-TKIs are more effective for NSCLC with activating mutations of $E G F R$ than for NSCLC with wild-type $E G F R$ (8). Among the known EGFR gene mutations, L858R and exon 19 deletion (EGFR 19del) account for the majority (85-90\%) of mutations in the EGFR kinase domain that respond well to EGFR-TKIs (9). However, not all EGFR mutations are sensitive to EGFRTKIs. The insertion of three or more nucleotides into exon 20 of $E G F R$ (EGFR ex20ins) is a known driver for NSCLC. Most EGFR ex20ins mutations are resistant to the currently available EGFR-TKIs and associated with a poor prognosis $(10,11)$. For example, it has been reported that the response rates of patients with $E G F R$ ex20ins to first-generation (erlotinib and gefitinib) and second-generation (afatinib) EGFR-TKIs are low, ranging from 0-8.7\% $(12,13)$. Although new drugs targeting NSCLC with EGFR ex20ins mutations, such as poziotinib, TAK-788 and TAS6417, have been developed, these agents are still being evaluated in clinical trials (14-16). Thus, effective treatment options for $E G F R$ ex20ins mutations are limited.

$E G F R$ ex20ins is usually mutually exclusive from certain other disease-driving gene mutations (such as EGFR 19del, $E G F R$ L858R, and mutations in ERBB2, ALK, BRAF and $R E T$ ) but is often accompanied by mutations in TP53, EGFR amplification, $C D K N 2 A$ and $C D K N 2 B$ (17). Among the many known $E G F R$ ex20ins mutation subtypes, patients with EGFR p.A763_Y764insFQEA respond to EGFR-
TKIs (18), and this has driven research into identifying other EGFR ex20ins mutation subtypes that are sensitive to EGFR-TKIs. However, the reality is that clinical outcomes will likely differ between two patients with the same $E G F R$ ex20ins mutation and the same treatment protocol, in part because of the influence of other accompanying gene mutations. Although EGFR amplification is present in $22 \%$ of patients with $E G F R$ ex20ins (17), no previous studies have evaluated the effect of $E G F R$ amplification on the clinical outcomes of patients with $E G F R$ ex20ins mutations treated with EGFR-TKIs or/and chemotherapy.

The aim of this study was to investigate whether EGFR amplification affects the progression-free survival (PFS) and overall survival (OS) of patients with EGFR ex20ins mutations treated with an EGFR-TKI and/or other chemotherapeutic agent. We present the following article in accordance with the STROBE reporting checklist (available at http://dx.doi.org/10.21037/jtd-20-1630).

\section{Methods}

\section{Study design and participants}

This is an observational longitudinal cohort study. This prospective case series included consecutive treatmentnaïve adult patients with advanced NSCLC who were seen at Guangdong Provincial People's Hospital between November 2017 and February 2019. The participants were enrolled from a prospectively managed database that also collected information regarding the patients' clinical characteristics, NGS data, treatment and clinical outcomes. The inclusion criteria were: (I) age $\geq 18$ years; (II) diagnosis of advanced NSCLC; (III) no previous treatment for NSCLC; and (IV) EGFR ex20ins mutation subsequently confirmed by NGS of peripheral blood and/ or tissue specimens. Patients with incomplete or missing data were excluded from the final analysis. The study investigators played no role in the design or implementation of the treatment protocols. The study was conducted in accordance with the Declaration of Helsinki (as revised in 2013). The study was approved by the Ethics and Scientific Committees of Guangdong Provincial People's Hospital (GDREC2016175H) and informed consent was taken from all the patients.

\section{Grouping}

The distribution of the EGFR ex20ins mutation subtypes 
was analyzed for all patients. Patients were divided into an $E G F R$ amplification group and a non-EGFR amplification group. Since the presence/absence of EGFR amplification could only be determined by NGS of tumor tissue, the clinical characteristic and the survival analysis (PFS and OS) only included patients who provided tumor tissue samples. Furthermore, patients were excluded from the survival analysis if they had EGFR mutations known to be sensitive to EGFR-TKIs (19del, L858R, G719X, L861Q and S768I). For the survival analysis, TKI subgroup including patients with once or more TKI treatment and Chemotherapy subgroup including patients with once or more chemotherapy treatment.

\section{Genomic analysis}

NGS was performed by Burning Rock Biotech (Guangzhou, China), which is a Clinical Laboratory Improvement Amendments-certified testing center. The samples for NGS included plasma and tumor tissue. For the extraction of cellfree DNA (cfDNA) from plasma, $10 \mathrm{~mL}$ of whole blood was collected in K3EDTA-containing tubes (Cell-Free DNA BCT; Streck, La Vista, NE, USA) and centrifuged at 2,000 g for $10 \mathrm{~min}$ at $4{ }^{\circ} \mathrm{C}$ within $72 \mathrm{~h}$ of collection. The supernatant was transferred to a $15-\mathrm{mL}$ centrifuge tube and centrifuged at $16,000 \mathrm{~g}$ for $10 \mathrm{~min}$ at $4{ }^{\circ} \mathrm{C}$, and the supernatant was transferred to a new tube for storage at $-80{ }^{\circ} \mathrm{C}$ until further use. Circulating cfDNA was extracted using the QIAamp Circulating Nucleic Acid kit (Qiagen, Hilden, Germany) and quantified using the dsDNA HS Assay Kit and Qubit 2.0 Fluorometer (Life Technologies, Carlsbad, CA, USA). For plasma genotyping, NGS of peripheral white blood cells was also performed to avoid false positive plasma genotyping due to clonal hematopoiesis. The extraction of DNA from tissue samples was achieved using the QIAamp DNA FFPE Tissue Kit (Qiagen) in accordance with the manufacturer's instructions. The DNA concentration was determined using the Qubit dsDNA Assay (Life Technologies).

Samples were analyzed using panels of 31, 61, 295 or 520 cancer-related genes. Burrows-Wheeler Aligner 0.7.10 was used to map the sequencing data (average sequencing depth, 10,000X). Local alignment optimization, variant calling and annotation (minimum locus depth, 100) were carried out using GATK 3.2, MuTect (Broad Institute, Cambridge, MA, USA) and VarScan (Genome Institute,
Washington University, USA). Insertions/deletions and single nucleotide variations (SNVs) were verified using at least two and eight supporting reads, respectively. Singlenucleotide polymorphisms (variants with a population frequency $>0.1 \%$ ) were excluded from further analysis, and the remaining variants were annotated with ANNOVAR and SnpEff v3.6. TopHat2 (Center for Computational Biology, Johns Hopkins University and Genome Sciences Department, University of Washington, USA) and Factera 1.4.3 were utilized for DNA translocation analysis.

Gene copy number variation (CNV) was evaluated with in-house scripts of tumor tissues. Coverage data were corrected for sequencing bias arising from GC content and probe design. The coverage of the various samples was normalized to comparable scales using the average coverage of all captured regions. Gene copy number $(\mathrm{CN})$ at each capture interval was calculated from the ratio of the coverage depth in detected circulating tumor DNA to the average coverage of $\geq 50$ reference samples without $\mathrm{CNV}$. CNV was determined using two criteria: (I) coverage of $>60 \%$ capture intervals of the genes differed significantly from reference samples $(\mathrm{P}<0.005$ for hotspot genes and $\mathrm{P}<0.001$ for other genes, $\mathrm{z}$-test comparing the coverage of each capture interval to the mean coverage of the interval in all control samples); (II) $\mathrm{CN}$ attained the minimal threshold for gain ( $>2.25$ for hotspot genes and $>2.5$ for others) or loss ( $<1.75$ for hotspot genes and $<1.5$ for others). $\mathrm{CNV}$ distribution plots were utilized to differentiate amplifications from polysomies. EGFR amplification was defined as $>2.75$ in the 520 panel and $>2.25$ in the other panels (19).

\section{Collection of clinical data}

The following clinical data were extracted from the database: sex, age, smoking history, tumor pathological type, treatment history and the date of occurrence of tumor progression or death.

\section{Outcomes}

All patients were followed-up until death or January 8 , 2020, whichever occurred first, and the median follow-up time was 630 days. OS was defined as the period from the initial systemic treatment to death of the patient due to any cause. PFS was defined as the period from the initiation 
treatment to progression of the tumor or death.

\section{Statistical analysis}

The data were analyzed using SPSS 22.0 (IBM Corp., Armonk, NY, USA) and Prism 7.0 (GraphPad, San Diego, CA, USA). Quantitative data are expressed as median (range) and were compared between groups using the MannWhitney $\mathrm{U}$ test. Count data are expressed as $n(\%)$ and were compared between groups using Fisher's exact test. Survival was analyzed using the Kaplan-Meier method and log-rank test. All statistical tests were two-sided, and $\mathrm{P}<0.05$ was considered statistically significant.

\section{Results}

\section{Clinical characteristics of the study participants}

A total of 39 patients were included in the EGFR ex20ins mutation subtypes analysis. For the clinical characteristic analysis, 8 patients were excluded because NGS of tumor tissue samples was not performed. For the survival analysis, 3 patients were excluded because they also had EGFRTKI-sensitive EGFR mutations (two carrying L858R and one carrying G719X), and 2 patients were excluded due to missing data. Therefore, a total of 26 patients were included in the overall survival analysis (Figure 1). As shown in Table 1, there were no significant differences between the EGFR amplification group $(\mathrm{n}=12)$ and non-EGFR amplification group $(\mathrm{n}=19)$ in sex, age, smoking history or tumor pathological type. Twenty-six EGFR ex20ins patients included in survival analysis. There were no significant differences between the EGFR amplification group ( $\mathrm{n}=11$ ) and non-EGFR amplification group $(\mathrm{n}=15)$ in sex, age, smoking history or tumor pathological type were (Table S1). For the subgroup analyses, 24 of the 26 patients were treated with at least one EGFR-TKI (EGFR amplification group, $\mathrm{n}=11$; non-EGFR amplification group, $\mathrm{n}=13$ ), and 20 of the 26 patients were administered another type of chemotherapeutic agent (EGFR amplification group, $\mathrm{n}=9$; non-EGFR amplification group, $\mathrm{n}=11$ ). For each of these subgroup analyses, there were no significant differences between the EGFR amplification group and non-EGFR amplification group in sex, age, smoking history or tumor pathological type (Tables $S 2$ and $S 3$ ).

\section{Distribution of EGFR ex20ins mutation subtypes}

Fifteen different EGFR ex20ins mutation subtypes were identified in the 39 patients (Figure 2). The three most common EGFR ex20ins subtypes were p.A767_D770dup (25.6\%), p.S768_D770dup (23.1\%) and p.N771_H773dup (10.3\%).

\section{Effects of EGFR amplification on the OS and PFS of patients carrying EGFR ex20ins mutations}

In the overall analysis, there were no significant differences between the EGFR amplification group $(\mathrm{n}=11)$ and nonEGFR amplification group ( $\mathrm{n}=15)$ in median OS (715 vs. 452 days, $\mathrm{P}=0.912$; Figure 3). Among the 20 patients who received at least one chemotherapeutic agent, there were no significant differences between the EGFR amplification group $(\mathrm{n}=9)$ and non- $E G F R$ amplification group $(\mathrm{n}=11)$ in median PFS (206 vs. 112 days, $\mathrm{P}=0.425$; Figure 4). Among the 24 patients administered at least one EGFR-TKI, the EGFR-TKI was used as a first-line treatment in 8 of the 11 patients $(72.7 \%)$ in the EGFR amplification group and 7 of the 13 patients $(53.8 \%)$ in the non-EGFR amplification group ( $\mathrm{P}=0.423$, Tables S2). Notably, median PFS was significant longer in the non-EGFR amplification group than in the EGFR amplification group (110 vs. 31 days, $\mathrm{P}=0.030$; Figure 5). Since the EGFRp.A763_Y764insFQEA mutation is sensitive to EGFR-TKIs (18), we repeated the analysis after excluding 2 patients carrying this mutation: median PFS remained significantly longer in the non-EGFR amplification group than in the EGFR amplification group (109 vs. 30.5 days, $\mathrm{P}=0.010$; Figure 6).

\section{Types of EGFR-TKIs used}

Among the 24 patients who received EGFR-TKIs, 5 patients were treated with afatinib, 2 patients were given erlotinib, 2 patients were administered osimertinib, 1 patient was treated with icotinib, 1 patient was given allitinib, and 13 patients were administered a new type of EGFR-TKI as part of a clinical trial (Figure 7). PFS 


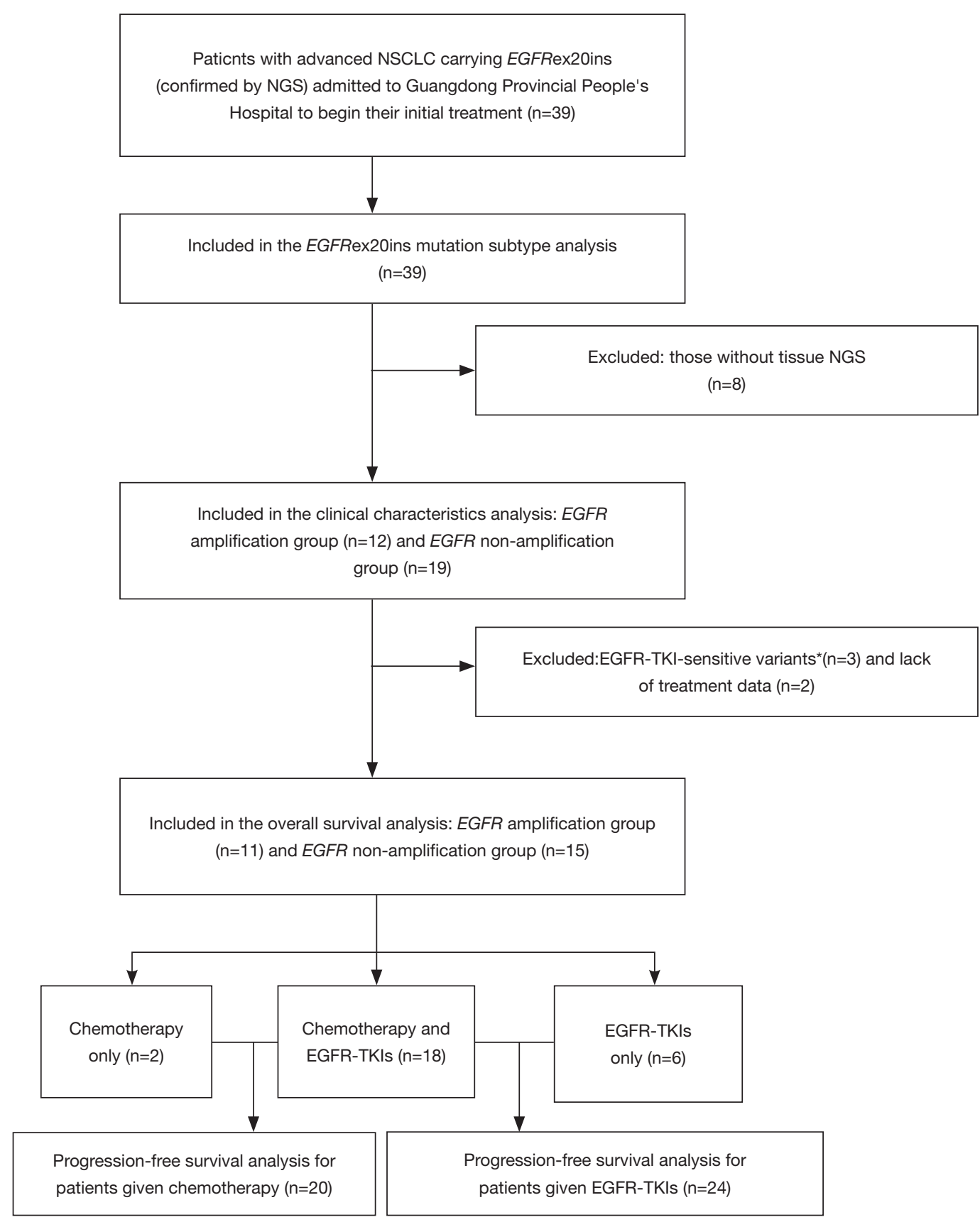

Figure 1 Flow diagram showing enrolment of the study participants. *Epidermal growth factor receptor (EGFR)-tyrosine kinase inhibitor (TKI)-sensitive mutations of EGFR included 19del, L858R, G719X, L861Q and S768I. EGFR-TKI, epidermal growth factor receptortyrosine kinase inhibitor; NGS, next-generation sequencing; NSCLC, non-small cell lung cancer.

exceeded 1 year in 2 patients: a 66-year-old male with stage IV lung cancer carrying the EGFRp.P772insH mutation who was administered afatinib as a first-line treatment
(PFS of 499 days) and a 64-year-old female with stage IV lung cancer carrying the EGFRp.P772_H773dup mutation who was administered a novel EGFR-TKI as a first-line 
Table 1 Clinical characteristics of 31 patients with EGFR ex20ins mutations

\begin{tabular}{lll}
\hline Characteristic & Non-EGFR amplification group $(\mathrm{n}=19)$ & EGFR amplification group $(\mathrm{n}=12)$ \\
\hline Age (years), median (range) & $60(34-80)$ & $55.5(38-67)$ \\
Sex, $\mathrm{n}(\%)$ & & \\
Female & $8(42.1)$ & $8(66.7)$ \\
Male & $11(57.9)$ & $4(33.3)$ \\
Smoking history, $\mathrm{n}(\%)$ & & \\
Smoker & $7(36.8)$ & $2(16.7)$ \\
Non-smoker & $12(63.2)$ & $10(83.3)$ \\
Tumor pathological type, $\mathrm{n}(\%)$ & $18(94.7)$ & $11(91.7)$ \\
Adenocarcinoma & $1(5.3)$ & $1(8.3)$ \\
Other & & 1.000 \\
\hline
\end{tabular}

Data were compared between groups using the Mann-Whitney $U$ test (age) or Fisher's exact test (all other parameters).

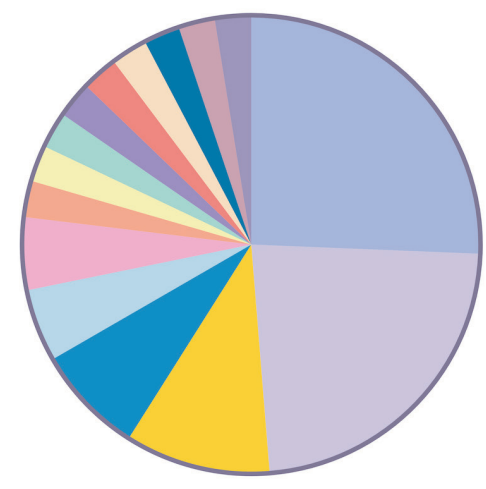

25.6\%p.A767_V769dup

23.1\%p.S768_D770dup

10.3\%p.N771_H773dup

7.7\%p.A763_Y764insFQEA

5.1\%p.H773dup

5.1\%p.P772_H773dup

2.6\%p.D770_N771insG

2.6\%p.D770 N771insGF

2.6\% p.D770delinsNNPH

$2.6 \%$ p.H773_V774insGNPH

2.6\%p.H773delinsPHPN

2.6\%p.N771_P772insG

Total $=39$

2.6\%p.P772_H773insGHP

2.6\%p.v769_D770insAAQ

2.6\%p.N771dup

Figure 2 The 15 EGFR ex20ins mutation subtypes identified in the 39 patients with advanced non-small cell lung cancer. The three most common mutation subtypes were p.A767_D770dup (25.6\%), p.S768_D770dup (23.1\%) and p.N771_H773dup (10.3\%).

treatment (PFS of 478+ days). Neither of these two patients had co-occurring EGFR amplification.

\section{Discussion}

This study demonstrates the EGFR ex20ins gene profile and the impact of EGFR amplification on survival of patients with EGFR ex20ins-positive NSCLC. Patients with NSCLC exhibiting both EGFR ex20ins mutation and
EGFR amplification had a significantly shorter PFS than patients with NSCLC exhibiting EGFR ex20ins mutation without EGFR amplification when an EGFR-TKI was used as the treatment but not when another chemotherapeutic drug was used as the treatment. To our knowledge, this is the first report to analyze the association between $E G F R$ amplification and clinical outcome in patients with $E G F R$ ex20ins mutations treated with EGFR-TKIs.

There are many different EGFR ex20ins mutations, and 


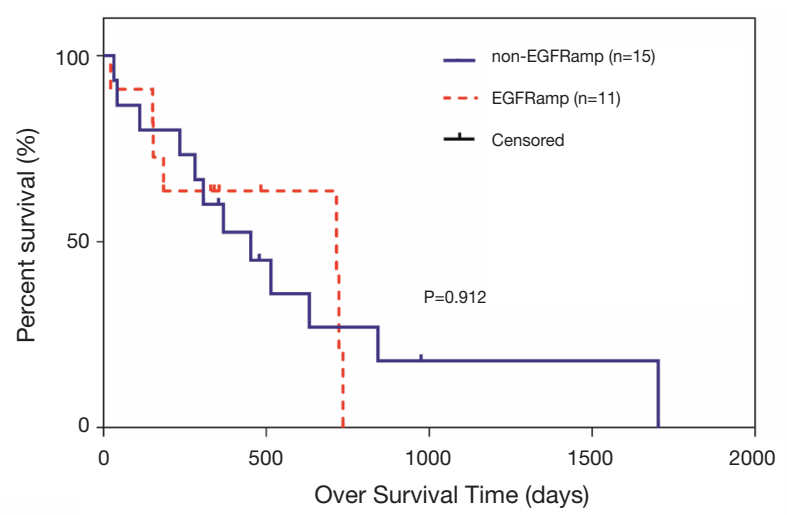

Figure 3 Overall survival of 26 patients with EGFR ex20ins mutations with or without EGFR amplification.

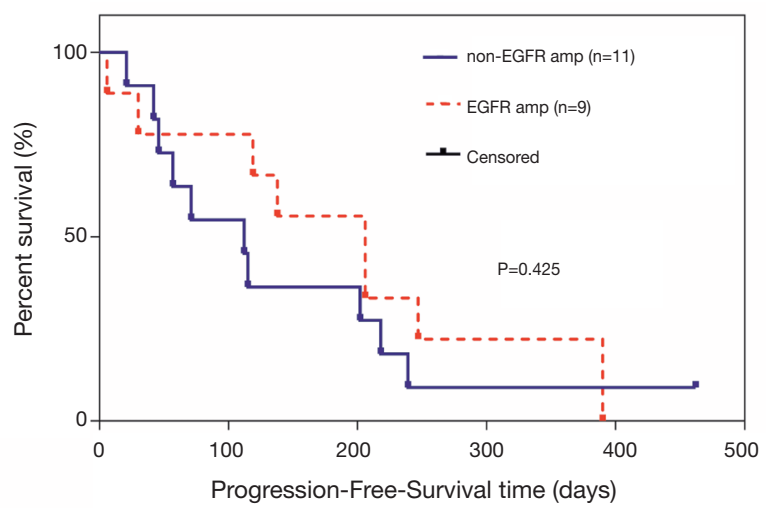

Figure 4 Progression-free survival of 20 patients with EGFR ex20ins mutations with or without EGFR amplification who were treated using chemotherapeutic agents.

a recent study of 14,483 NSCLC specimens from mainly Caucasian patients identified 64 EGFR ex20ins mutations (17). The three most common mutations detected in the above study were p.A767_D770dup (21\%), p.S768_D770dup (20\%) and p.N771_H773dup (8\%) (17), which is in good agreement with our findings. Although the overall rate of EGFR mutations in NSCLC may be higher in Chinese patients than in Caucasian patients, ethnicity appears to have little effect on the rates of the common EGFR ex20ins mutations.

Previous research has indicated that EGFR ex20ins mutations are generally mutually exclusive from certain other disease-driving genes such as mutations in ERBB2, $A L K, B R A F$ and RET as well as other EGFR mutations (17). However, EGFR ex20ins mutations are usually accompanied by $C D K N 2 A$ mutations and amplifications of TP53 and $E G F R$ (17). It is worth noting that the rate of $E G F R$ amplification in NSCLC carrying EGFR ex20ins mutations was only $22 \%$ in the study by Riess et al. (17) compared with $38.7 \%$ in our study. There are three possible explanations for the apparent difference between our findings and those of Riess et al.: (I) the study by Riess et al. included mainly Caucasian patients, who are known to have a lower EGFR mutation rate than Asian patients; (II) the sample size in the study by Riess et al. $(\mathrm{n}=263)$ was much larger than that of our study ( $\mathrm{n}=31)$; and (III) the NGS methods and definition of EGFR amplification differed between the two studies. With regard to the latter point, EGFR amplification was defined as $>6$ copy number $(\mathrm{CN})$ in the study by Riess et al. and as $\mathrm{CN}$ reaching the minimum threshold $(>2.75$ in the 520 panel and $>2.25$ in the other panels) in our study; this difference in definition may have contributed to the lower proportion of patients considered to have EGFR amplification in the study by Riess et al. It 


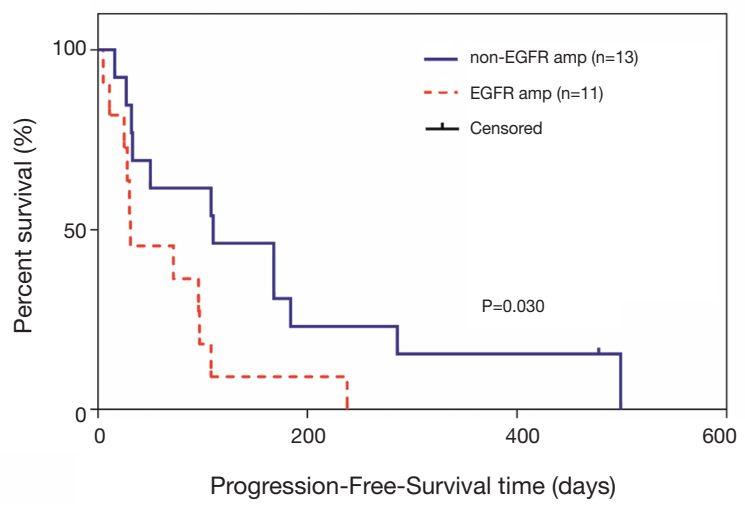

Figure 5 Progression-free survival of 24 patients with $E G F R$ ex20ins mutations with or without EGFR amplification who were treated using epidermal growth factor receptor-tyrosine kinase inhibitors.

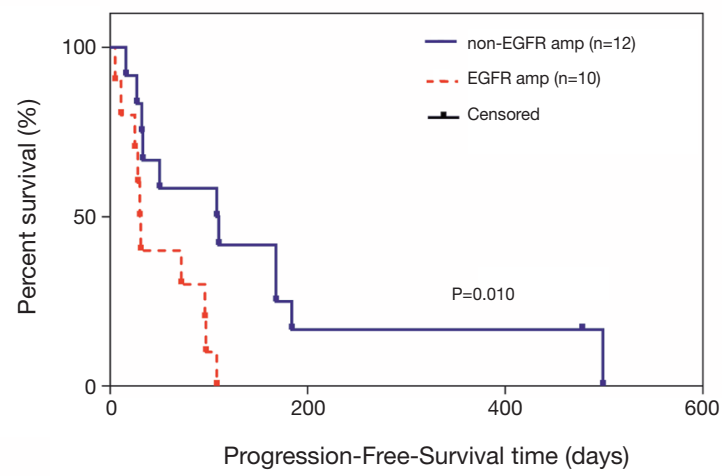

Figure 6 Progression-free survival of 22 patients with EGFR ex20ins mutations with or without EGFR amplification who were treated using epidermal growth factor receptor-tyrosine kinase inhibitors. Patients with the EGFR p.A763_Y764insFQEA mutation were excluded from the analysis.

should be noted that we have previously used fluorescence in situ hybridization (FISH) to validate the NGS methods used to detect EGFR amplification in this study (19). Since there are many NGS testing methods, each with its own definition of EGFR amplification, more research is needed to obtain a consensus threshold for the definition of $E G F R$ amplification.

This study found that PFS was significantly shorter in patients with EGFR amplification than in those without $E G F R$ amplification after their first treatment with an EGFR-TKI, suggesting a less favorable therapeutic effect in patients with $E G F R$ ex20ins mutations who also have EGFR amplification. However, EGFR amplification status did not have a significant effect on PFS in patients given their first chemotherapy. Previous reports have described an association between $E G F R$ amplification and the therapeutic effects of EGFR-TKIs and anti-EGFR monoclonal antibody (20-22). The therapeutic effect of an EGFRTKI is achieved mainly through inhibition of the EGFR signaling pathway, whereas the benefits of other types of chemotherapy may not be directly related to suppression of EGFR signaling. Therefore, EGFR amplification may only attenuate the therapeutic effects of drugs that block the EGFR signaling pathway.

Anti-EGFR monoclonal antibody can inhibit EGFR signaling, and its combination with cetuximab may be a good treatment option for patients with EGFR ex20ins mutation with $E G F R$ amplification. In a study of 4 patients carrying EGFR ex20ins mutations, the combination of afatinib with cetuximab achieved a partial response in 2 


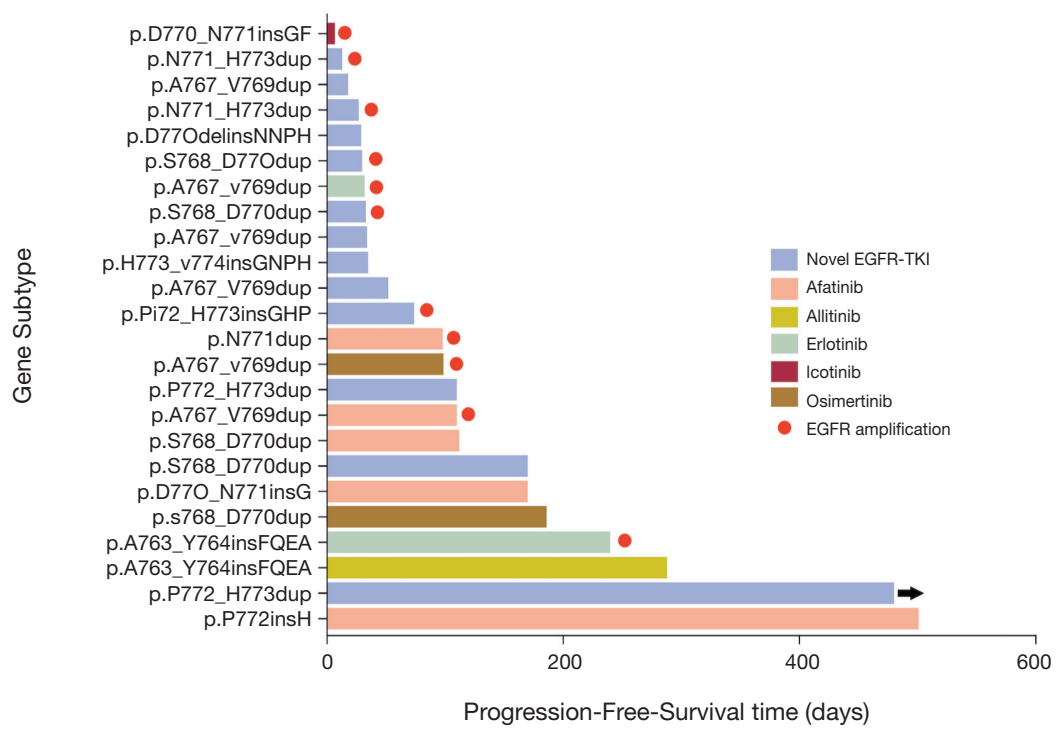

Figure 7 Progression-free survival of patients with different EGFR ex20ins mutations with or without EGFR amplification who were treated using various epidermal growth factor receptor-tyrosine kinase inhibitors (pink blue: novel agent; pink: afatinib; dark green: allitinib; light green: erlotinib; dark purple: icotinib; brown: osimertinib). Red dots represent patients with EGFR amplification. Black arrow represents that until the end date of the follow-up, the patient's treatment is considered effective and continued.

of 3 patients with EGFR CN $1-2$ and in another patient with EGFR CN 3-4, and PFS was 2.7, 4.4, 6.4+ (treatment ongoing) and 17.6 months for these patients (23). These results indicate that the combination of an EGFR-TKI with cetuximab may achieve a better therapeutic effect in patients with NSCLC exhibiting both an EGFR ex20ins mutation and $E G F R$ amplification. Future therapeutic strategies for patients with EGFR ex20ins mutations will depend on the results of clinical trials of various new drugs. For now, our first priority is to decide how to optimize the treatment protocol using the drugs currently available on the market.

There are still many limitations in our study. First, the EGFR-TKI treatment regimens varied between patients. The overall efficacy of EGFR-TKIs were limited, and there were obvious differences in sensitivity between individuals. Second, this was a single-center study with a small sample size. Thus, our conclusion was just a suggestive and trending one and larger sample size studies are needed. Patient recruitment is ongoing to validate our preliminary findings.

In summary, there might be a tendency that EGFR amplification is associated with a poorer PFS in patients with EGFR ex20ins-positive NSCLC treated with EGFRTKIs. Screening for EGFR ex20ins and EGFR amplification with NGS might help to identify patients who would benefit from therapy with an EGFR-TKI. Further largescale clinical trials with better drugs targeting EGFR ex20ins are needed to confirm our findings.

\section{Acknowledgments}

The authors thank the patients, their families and the study personnel who participated in this trial.

Funding: This work was supported by the National Key R\&D Program of China (No. 2016YFC1303800 to Q Zhou), the National Natural Science Foundation of China (No. 81871891 to Q Zhou), the High-level Hospital Construction Project (No. DFJH201810 to Q Zhou) and Key Lab System Project of Guangdong Science and Technology Department-Guangdong Provincial Key Lab of Translational Medicine in Lung Cancer (Grant No. 2017B030314120, to YL WU).

\section{Footnote}

Reporting Checklist: The authors have completed the STROBE reporting checklist. Available at http://dx.doi. org/10.21037/jtd-20-1630 
Data Sharing Statement: Available at http://dx.doi. org/10.21037/jtd-20-1630

Conflicts of Interest: All authors have completed the ICMJE uniform disclosure form (available at http://dx.doi. org/10.21037/jtd-20-1630). QZ reports speaker fees from AstraZeneca, and Roche. YLW reports speaker fees from AstraZeneca, Eli Lilly, Pfizer, Roche, and Sanofi. WZZ reports speaker fees from AstraZeneca and Roche. The other authors have no conflicts of interest to declare.

Ethical Statement: The authors are accountable for all aspects of the work in ensuring that questions related to the accuracy or integrity of any part of the work are appropriately investigated and resolved. The study was conducted in accordance with the Declaration of Helsinki (as revised in 2013). This study was approved by the Ethics and Scientific Committees of Guangdong Provincial People's Hospital (GDREC2016175H) and informed consent was taken from all the patients.

Open Access Statement: This is an Open Access article distributed in accordance with the Creative Commons Attribution-NonCommercial-NoDerivs 4.0 International License (CC BY-NC-ND 4.0), which permits the noncommercial replication and distribution of the article with the strict proviso that no changes or edits are made and the original work is properly cited (including links to both the formal publication through the relevant DOI and the license). See: https://creativecommons.org/licenses/by-nc-nd/4.0/.

\section{References}

1. Siegel RL, Miller KD, Jemal A. Cancer statistics, 2019. CA Cancer J Clin 2019;69:7-34.

2. Barta JA, Powell CA, Wisnivesky JP. Global Epidemiology of Lung Cancer. Ann Glob Health 2019;85:8.

3. Molina JR, Yang P, Cassivi SD, et al. Non-small cell lung cancer: epidemiology, risk factors, treatment, and survivorship. Mayo Clin Proc 2008;83:584-94.

4. Beadsmoore CJ, Screaton NJ. Classification, staging and prognosis of lung cancer. Eur J Radiol 2003;45:8-17.

5. Loong HH, Kwan SS, Mok TS, et al. Therapeutic strategies in EGFR mutant non-small cell lung cancer. Curr Treat Options Oncol 2018;19:58.

6. Yang JC, Ahn MJ1, Kim DW, et al. Osimertinib in pretreated T790M-positive advanced non-small- cell lung cancer: AURA study phase II extension component. J Clin
Oncol 2017;35:1288-96.

7. Mok TS, Wu YL, Thongprasert S, et al. Gefitinib or carboplatin-paclitaxel in pulmonary adenocarcinoma. $\mathrm{N}$ Engl J Med 2009;361:947-57.

8. Zhao D, Chen X, Qin N, et al. The prognostic role of EGFR-TKIs for patients with advanced non-small cell lung cancer. Sci Rep 2017;7:40374.

9. Gazdar AF. Activating and resistance mutations of EGFR in non-small-cell lung cancer: role in clinical response to EGFR tyrosine kinase inhibitors. Oncogene 2009;28:S24-S31.

10. Arcila ME, Nafa K, Chaft JE, et al. EGFR exon 20 insertion mutations in lung adenocarcinomas: prevalence, molecular heterogeneity, and clinicopathologic characteristics. Mol. Cancer Ther 2013;12:220-9.

11. Kobayashi Y, Mitsudomi T. Not all epidermal growth factor receptor mutations in lung cancer are created equal: Perspectives for individualized treatment strategy. Cancer Sci 2016;107:1179-86.

12. Yang JC, Sequist LV, Geater SL, et al. Clinical activity of afatinib in patients with advanced non-small-cell lung cancer harbouring uncommon EGFR mutations: a combined post-hoc analysis of LUX-Lung 2, LUX-Lung 3, and LUX-Lung 6. Lancet Oncol 2015;16:830-8.

13. Tu HY, Ke EE, Yang JJ, et al. A comprehensive review of uncommon EGFR mutations in patients with non-small cell lung cancer. Lung Cancer 2017;114:96-102.

14. Robichaux JP, Elamin YY, Tan Z, et al. Mechanisms and clinical activity of an EGFR and HER2 exon 20-selective kinase inhibitor in non-small cell lung cancer. Nat Med 2018;24:638-46.

15. Doebele RC, Riely GJ, Spira AI, et al. First report of safety, PK, and preliminary antitumor activity of the oral EGFR/HER2 exon 20 inhibitor TAK-788 (AP32788) in non-small cell lung cancer (NSCLC). J Clin Oncol 2018;36:9015.

16. Hasako S, Terasaka M, Abe N, et al. TAS6417, a novel EGFR inhibitor targeting exon 20 insertion mutations. Mol Cancer Ther 2018;17:1648-58.

17. Riess JW, Gandara DR, Frampton GM, et al. Diverse EGFR exon 20 insertions and co-occurring molecular alterations identified by comprehensive genomic profiling of non-small cell lung cancer. J Thorac Oncol 2018;13:1560-8.

18. Lin YT, Liu YN, Wu SG, et al. Epidermal growth factor receptor tyrosine kinase inhibitor-sensitive exon 19 insertion and exon 20 insertion in patients with advanced non-small-cell lung cancer. Clin Lung Cancer 
2017;18:324-332.e1.

19. Zhang YC, Chen ZH, Zhang XC, et al. Analysis of resistance mechanisms to abivertinib, a third-generation EGFR tyrosine kinase inhibitor, in patients with EGFR T790M-positive non-small cell lung cancer from a phase I trial. EBioMedicine 2019;43:180-7.

20. Paz-Ares L, Socinski MA, Shahidi J, et al. Correlation of EGFR-expression with safety and efficacy outcomes in SQUIRE: a randomized, multicenter, open-label, phase III study of gemcitabine-cisplatin plus necitumumab versus gemcitabine-cisplatin alone in the first-line treatment of patients with stage IV squamous non-small-cell lung cancer. Ann Oncol 2016;27:1573-9.

Cite this article as: Gao X, Wei XW, Zheng MY, Chen ZH, Zhang XC, Zhong WZ, Yang JJ, Wu YL, Zhou Q. Impact of EGFR amplification on survival of patients with EGFR exon 20 insertion-positive non-small cell lung cancer. J Thorac Dis 2020;12(10):5822-5832. doi: 10.21037/jtd-20-1630
21. Wang C, Xu F, Shen J, et al. Successful treatment of lung adenocarcinoma with gefitinib based on EGFR gene amplification. J Thorac Dis 2018;10:E779-E783.

22. Hirsch FR, Varella-Garcia M, McCoy J, et al. Increased epidermal growth factor receptor gene copy number detected by fluorescence in situ hybridization associates with increased sensitivity to gefitinib in patients with bronchioloalveolar carcinoma subtypes: a Southwest Oncology Group Study. J Clin Oncol 2005;23:6838-45.

23. van Veggel B, de Langen AJ, Hashemi SMS, et al. Afatinib and cetuximab in four patients with EGFR Exon 20 insertion-positive advanced NSCLC. J Thorac Oncol 2018;13:1222-6. 


\section{Supplementary}

Table S1 Clinical characteristics of 26 patients with EGFR ex20ins mutations included in the survival analysis

\begin{tabular}{lll}
\hline Characteristic & Non-EGFR amplification group $(\mathrm{n}=15)$ & EGFR amplification group $(\mathrm{n}=11)$ \\
\hline Age (years), median (range) & $56(34-72)$ & $55(38-65)$ \\
Sex, $\mathrm{n}(\%)$ & & \\
Female & $5(33.3 \%)$ & $8(72.7 \%)$ \\
Male & $10(66.7 \%)$ & $3(27.3 \%)$ \\
Smoking history, $\mathrm{n}(\%)$ & & \\
Smoker & $7(46.7 \%)$ & $1(9.1 \%)$ \\
Non-smoker & $8(53.3 \%)$ & $10(90.9 \%)$ \\
Tumor pathological type, $\mathrm{n}(\%)$ & & 10.111 \\
Adenocarcinoma & $14(93.3 \%)$ & $10(90.9 \%)$ \\
Other & $1(6.7 \%)$ & $1(9.1 \%)$ \\
\hline
\end{tabular}

Data were compared between groups using the Mann-Whitney U test (age) or Fisher's exact test (all other parameters).

Table S2 Clinical characteristics of patients with EGFR ex20ins mutations included in the subgroup analysis of progression-free survival for patients treated with epidermal growth factor receptor-tyrosine kinase inhibitors

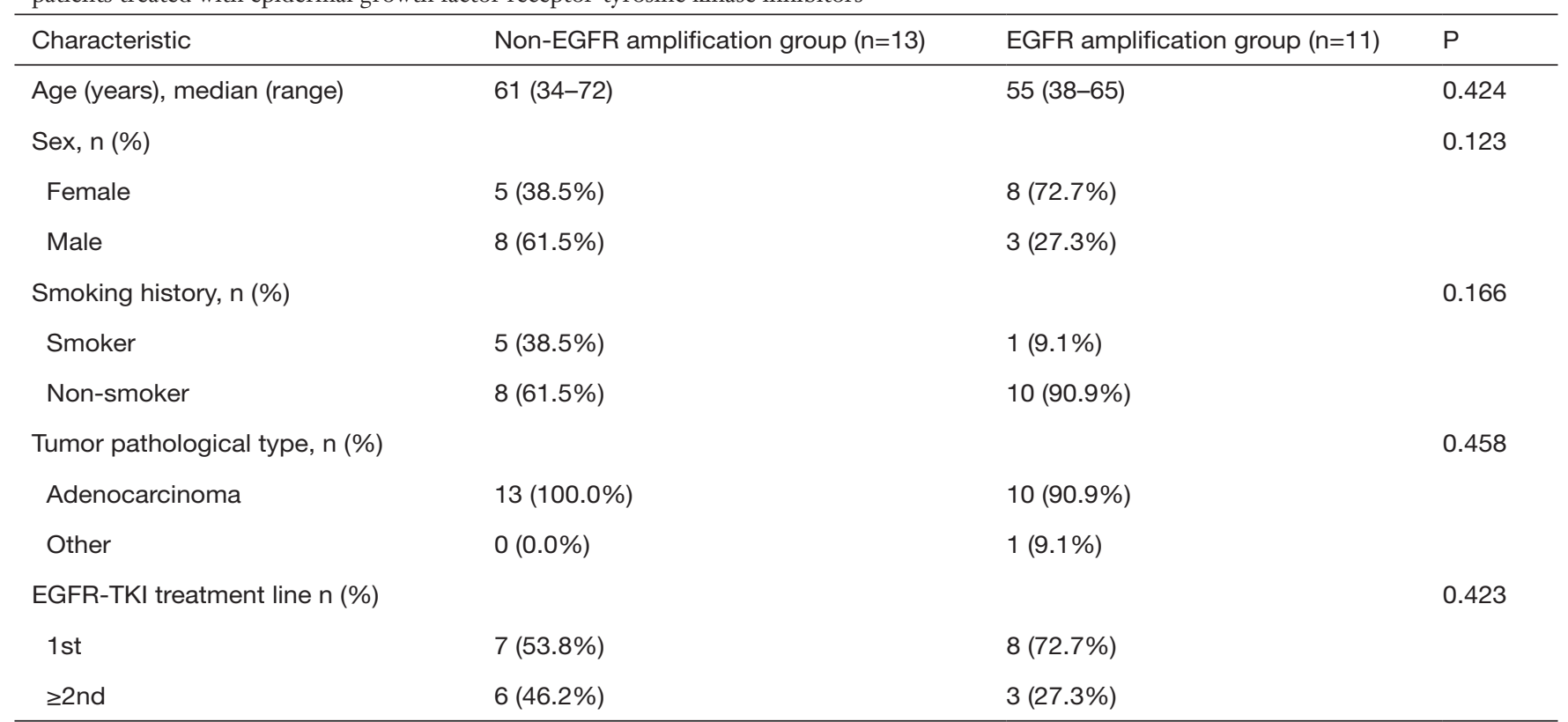

Data were compared between groups using the Mann-Whitney $U$ test (age) or Fisher's exact test (all other parameters). EGFR-TKI, epidermal growth factor receptor-tyrosine kinase inhibitor. 
Table S3 Clinical characteristics of patients with EGFR ex20ins mutations included in the subgroup analysis of progression-free survival for patients treated with chemotherapeutic agents other than epidermal growth factor receptor-tyrosine kinase inhibitors

\begin{tabular}{|c|c|c|c|}
\hline Characteristic & Non-EGFR amplification group $(n=11)$ & EGFR amplification group $(n=9)$ & $\mathrm{P}$ \\
\hline Sex, n (\%) & & & 0.175 \\
\hline Female & $3(27.3 \%)$ & $6(66.7 \%)$ & \\
\hline Male & $8(72.7 \%)$ & $3(33.3 \%)$ & \\
\hline Smoker & $6(54.5 \%)$ & $1(11.1 \%)$ & \\
\hline Non-smoker & $5(45.5 \%)$ & $8(88.9 \%)$ & \\
\hline Tumor pathological type, n (\%) & & & 1.000 \\
\hline Adenocarcinoma & $10(90.9 \%)$ & $8(88.9 \%)$ & \\
\hline $1 \mathrm{st}$ & $8(72.7 \%)$ & $2(22.2 \%)$ & \\
\hline$\geq 2$ nd & $3(27.3 \%)$ & $7(77.8 \%)$ & \\
\hline
\end{tabular}

Data were compared between groups using the Mann-Whitney $U$ test (age) or Fisher's exact test (all other parameters). 